\title{
AFM as a tool to probe and manipulate cellular processes
}

\author{
Charles-Antoine Lamontagne • Charles M. Cuerrier • \\ Michel Grandbois
}

Received: 12 October 2007 / Revised: 23 November 2007 / Accepted: 27 November 2007 / Published online: 15 December 2007

(C) Springer-Verlag 2007

\begin{abstract}
The exploration of molecular processes governing physiological functions has significantly benefited from the emergence of novel nanoscaled techniques. Atomic force microscopy in force measurement mode can be used to investigate a multitude of cellular events in individual living cells with great sensitivity. Precise regions of the plasma membrane can be examined in relation to particular signalling pathways activated by a mechanical stimulus. Similarly, subtle cellular movements induced by biochemical activation of specific membrane receptors can be detected in real time with excellent temporal and spatial resolution. The possibility to challenge locally and mechanically cell surface receptors also provides information on the control exerted by a cell over individual adhesion sites. Overall, this information is vital for an in-depth understanding of mechanisms related to cellular movement and morphological regulation. Lastly, atomic force microscope-based nanomanipulations on living cells have recently been proposed as a tool to influence and monitor cellular homeostasis by introducing specific molecular entities into or extracting them from the cytoplasm of individual cells. This review provides detailed examples on how such atomic force microscopy experiments can be conducted to investigate processes relevant to cell physiology.
\end{abstract}

Keywords AFM · Cell mechanics · Cell signalling · Functionalised AFM tips · Molecular interaction . Transfection

C.-A. Lamontagne $\cdot$ C. M. Cuerrier $\cdot$ M. Grandbois $(\triangle)$ Département de Pharmacologie, Faculté de Médecine, Université de Sherbrooke,

3001, 12e Ave. Nord,

Sherbrooke, QC, Canada

e-mail: michel.grandbois@usherbrooke.ca

\author{
Abbreviations \\ AFM atomic force microscope \\ AngII angiotensin II \\ HA hyaluronic acid \\ PKC protein kinase $\mathrm{C}$ \\ PMA phorbol myristate acetate \\ EGFP enhanced green fluorescent protein
}

\section{Introduction}

Over the past two decades, advances in instrumentation have greatly contributed to the increasing number of cellular and molecular biology tools available to investigate nanoscaled biomolecular structures. The application of atomic force microscopy (AFM) to biology has provided unique opportunities to visualise [1-5] and manipulate [5-12] functional biological structures with nanometre resolution in physiological conditions and has enabled researchers to address biological questions that could not otherwise be investigated. Force measurement with the AFM has become a tool of choice to probe and manipulate a wide variety of cellular events. Indeed, the opportunity to precisely address nanoscaled structures on individual cells and to measure forces in the piconewtons range has provided unprecedented mechanistic details of cellular processes. Moreover, the ability to attach functional biomolecules to the tip of the atomic force microscope has made it possible to characterise molecular recognition processes directly on the surfaces of living cells. This experimental approach has been widely applied to study cellular adhesion processes [13-30] and to map the distribution of membrane receptors, an application described in great details in several recent reviews [31-34]. 
AFM force experiments on living cells have also opened up new vistas in the investigation of the mechanical properties of individual cells and have demonstrated the importance of these mechanical properties to various physiological and physiopathological processes [17, 19, 35-40]. This review will discuss some recent contributions of the AFM to cell physiology with a focus on the probing and nanomanipulation of structures in living cell. We first describe the use of AFM to investigate the mechanical properties of living cells. We then present experiments in which the mechanical properties of individual cells can be modulated using external mechanical or biochemical signals involving the activation of cellular signalling pathways. We also address molecular events in dynamic cellular processes responsible for tissue cohesion and cell migration. In this context, AFM can be applied to characterise local cellular adhesion events involving a wide variety of molecular structures, including molecular adhesion pairs, the cell membrane and the underlying cytoskeleton. Lastly, the possibility of using AFM to insert molecular entities into the cytosol of individual cells to influence their internal biochemical processes will be discussed.

\section{Mechanical sensing of cellular processes}

The mechanical properties of biological tissues are normally assessed through mechanical experiments probing, in macroscopic samples, the parallel contribution of molecular and cellular components. Despite the wealth of information provided by such experiments, very little is known about the involvement of individual elements making up a given system. To date, very few experiments have been designed to evaluate the mechanical contributions of individual cells to specific physiological responses. The importance of the mechanical responses and behaviour of single cells is especially relevant in dynamic physiological processes such as tissue remodelling, cell motility and signal transduction in various mechanically sensitive cells such as those involved in hearing, bone remodelling and pressure sensing in vascular systems [36, 37, 39, 41-46]. Several new concepts, describing mechanical responses in cells, have emerged since the development of AFM-thanks to its ability to apply or record minute forces on individual cells. The force-distance curves obtained by the direct indentation of the cell surface with an AFM tip have been used to quantify the mechanical properties of cells in contexts relevant to physiological processes such as cell migration, contraction and division [37, 39, 40, 45]. The overall mechanical characteristics of a cell are largely determined by its plasma membrane, as well as by the different molecular components of the internal cytoskeleton and external glycocalix. Indeed, to preserve its integrity and adapt to its external environment, a cell modulates its cytoskeleton architecture, which is composed of actin filaments, microtubules and intermediate filaments. In addition, the mechanical characteristics of a cell are also sensitive to the lipid and protein content of the membrane, as well as their interactions with diverse components of the extracellular environment $[27,35,38,40,47-50]$. The possibility of characterising the mechanical properties of cells in real-time opens up new avenues for a more detailed understanding of physiological processes. Oberleithner et al. [51] recently monitored changes in endothelial cell stiffness caused by the stimulation of the aldosterone receptor and related this phenomenon to the endothelial dysfunction observed in hyperaldosteronism, a pathological condition associated with high blood pressure and fluid retention in organs. Inspired from cell surface indentation experiments, an emerging procedure consists in contacting the cell surface with the AFM probe and recording, in realtime, the fluctuations caused by membrane and cystosolic movements. In this recording mode, the time course of cellular activities leading to shape remodelling, movements, or contractile activities can be precisely monitored. For example, the contractile responses of myocytes and fibroblasts have been quantified using AFM [52-54]. In myocytes, it is possible to monitor acto-myosin-dependent pulsatile activity in individual cells with a transverse spatial resolution of several nanometres and a temporal resolution of the order of $100 \mu \mathrm{s}$ [52]. While these studies are a convincing application of AFM to cell mechanical activities, it should be noted that such correlated contractions can also be monitored by conventional optical microscopy. The quantification of small-amplitude fluctuations observed as a result of subtle metabolic-dependent remodelling in nonmuscle cells is a promising application of AFM in cell biology. Fluctuations in intracellular cytoskeletal structures at the protruding edge of motile cells have been observed using various optical techniques $[55,56]$. Although these activities can be optically detected, the quantification of nanoscaled events and their association with specific cellular mechanical activities is problematic, mainly because of the low resolution of the optical approaches. In this context, AFM has recently been used to monitor membrane fluctuations in living Saccharomyces cerevisiae cells. These nanoscaled fluctuations are associated with the temperature and metabolic activity of the cells $[57,58]$. Moreover, AFM allows for real-time, local monitoring of membrane fluctuations at the location on the cell surface that is undergoing extensive cytoskeleton movement and remodelling. The mechanical fluctuations recorded at the protruding edge of individual cells are larger in amplitude than those recorded in other regions of the cells, indicating that the cytoskeleton at the protruding edge is in a greater dynamical state [37, $45,59]$. 
We recently extended the use of AFM to the local monitoring of angiotensin II (AngII)-induced mechanical responses (Fig. 1). AngII, a hormone involved in vascular regulation, activates $\mathrm{AT}_{1}$ receptors at the surface of endothelial and smooth muscle cells. Activation of the $\mathrm{AT}_{1}$ receptor induces various signalling pathways leading to actin remodelling and contraction [60] in physiological processes relevant to biological fluids transport and cell monolayer permeability. Figure $1 \mathrm{~b}$ shows a typical mechanical response recorded with the AFM where the signal is expressed in terms of cell membrane displacement as a function of time. The main feature of the AngII-induced mechanical response is the large upward displacement of the membrane (Fig. 1b). Selective inhibitors of the related signalling pathways were used to attribute this movement to acto-myosin-dependent contractile activity [60]. Another important feature in the mechanical signal is the presence of smaller random fluctuations after the initial main contractile response. These nanoscaled height fluctuations reflect the structural changes occurring within the cell that are associated with increased cytoskeletal activity [compare the signal before (1) and after AngII stimulation (3) in Fig. 1b]. It is important to note that these nanometric contractions and cell membrane fluctuations, which are not easily detected using conventional optical techniques, may be the result of subtle mechanisms that modulate the cohesion and activity of various cellular networks.

Like agonist-based stimulation, certain cells can be activated after the application of a local mechanical stimulus.
Cell responses to mechanical stimulations have been studied in various cellular contexts using AFM [41, 42, 61]. By measuring intra-cellular calcium mobilisation in osteoblasts, Charras and Horton demonstrated that the mechanical stimulation of individual cells can propagate to neighbouring cells [62]. Obtaining such information relies on the ability to apply a mechanical stimulation to a single cell. The mechanistic details obtained through experiments on individual living cells will improve our understanding of the regulation of macroscopic physiological processes.

\section{AFM probing of adhesion behaviour in living cells}

The capacity of cells to adhere to components of the extracellular matrix and neighbouring cells is largely responsible for tissue cohesion. Dynamic regulation of adhesion and deadhesion processes enable tissue remodelling and cell migration. These interactions are fundamental to numerous physiological and physiopathological processes. A large variety of adhesion sites on cell surfaces that ensure the transmission of forces between the cell and its environment have been identified. Nanomanipulating specific molecular recognition pairs on living cells provides valuable clues in the quest to understand cellular adhesion mechanisms. In the past decade, AFM in the force measurement mode has been used to characterise adhesion events at high spatial and force resolutions. The functionalisation of an AFM tip with specific chemical groups, biomolecules and even living cells has

\section{a}

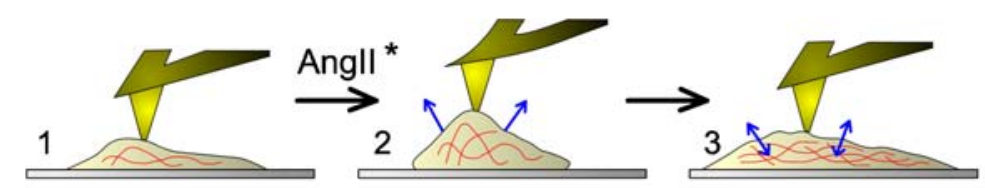

b

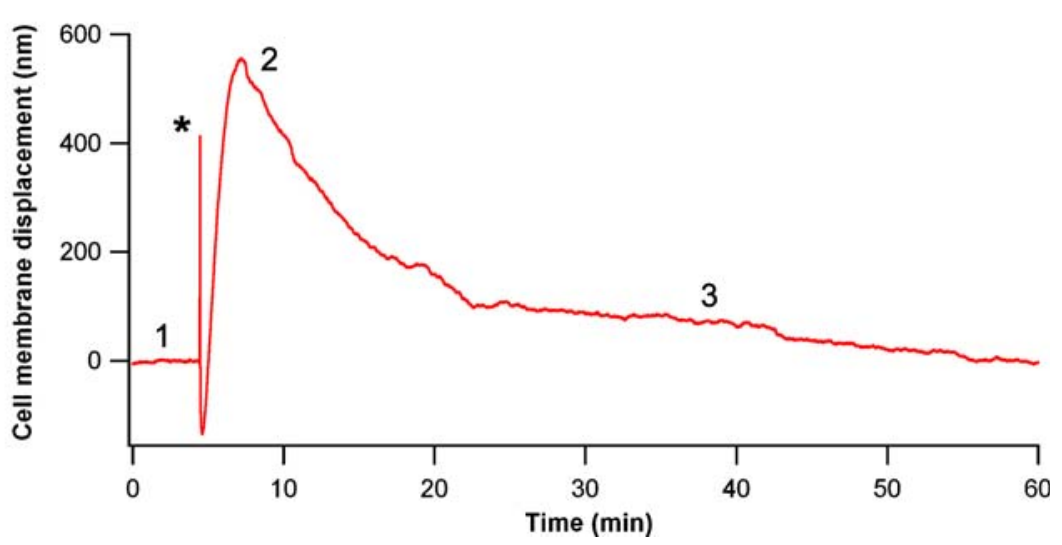

Fig. 1 Monitoring of cell mechanical fluctuations in AT1-transfected HEK-293 cells stimulated by AngII. a A schematic representation of a typical experiment where an AFM tip contacts the cell body with minimal force to record the mechanical response induced by AngII stimulation. b A mechanical response plotted in cell membrane displacement units (nanometer) as a function of time. A baseline is recorded for several minutes (1) before the injection of $100 \mathrm{nM}$ AngII (asterisk). A typical mechanical response is characterised by an initial contraction of the cell body (2) followed by small fluctuations in the amplitude of the signal (3) 
revealed how cells interact with various artificial and biological surfaces $[14,15,18-21,24,25,27,29]$. For example, Benoit et al. described the interaction of cells with diverse artificially tailored surfaces with the goal of guiding the rational design of medical implants [17]. Similar experiments have revealed molecular mechanisms that might explain the divergences observed in the rolling behaviours of leukocytes over different selectin-decorated surfaces [9, 22]. AFM experiments have also characterised specific cell signalling pathways affecting fibronectin-mediated adhesion in the gastrulation process in the early development of zebrafish embryos [26]. In addition, AFM has been employed to directly measure interaction forces between living cells $[13,16,17,23,28,63]$. In the Dictyostelium model of cell aggregation, two different types of adhesions have been observed depending on the developmental stage of the cells. In this system, the relative contribution of csA adhesion proteins was assessed through genetic manipulations and variations of the composition of cellular environments $[16,17]$. Thie et al. used AFM to examine the preferential interaction of human trophoblasts with uterine epithelium cells. Their study indicated that there is a weak initial cell-cell interaction and that molecular events occur that lead to strong bond formation and successful implantation of the embryo [13]. Comparable experiments were conducted by Zhang et al. [23], who observed that binding occurred between leukaemia and endothelial cells during cell transmigration. Their study, which showed that leukaemia cells preferentially bind to the border of adjacent endothelial cells, demonstrated the need for tools capable of probing molecular events in specific locations on the cell surface. This is particularly important for cellular motility and invasion processes, which strongly rely on focalised distribution of adhesion sites, as well as on the activation state of the adhesion molecules [64].

Functionalisation of the AFM tip with specific ligands for cell membrane adhesion receptors allows for the precise positioning of a molecular probe over a selected region of the cell surface. This kind of experiment provides information on the spatial regulation and contribution of subcellular structures to the adhesion behaviour of cells for particular molecular substrates. In Fig. 2, we present a typical experiment where AFM was used to probe ligand-receptor interactions on glioblastoma cells in relation to their activation state and the localisation of adhesion receptors [30]. The tip was functionalised with hyaluronic acid (HA), a

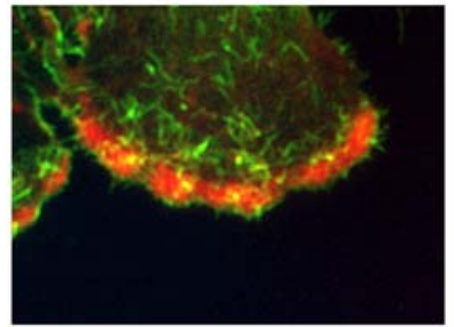

e

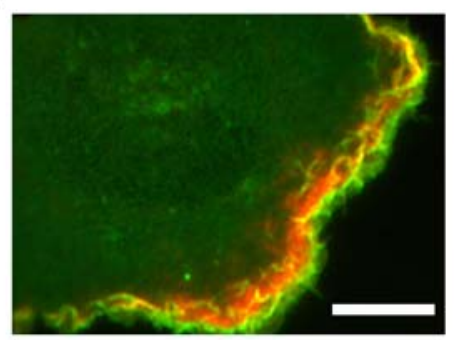

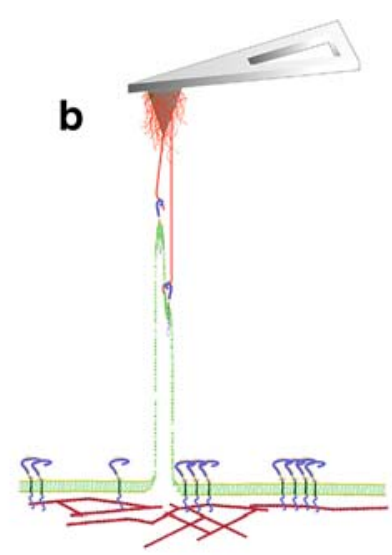

f

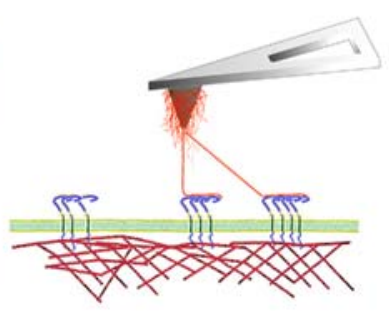

C

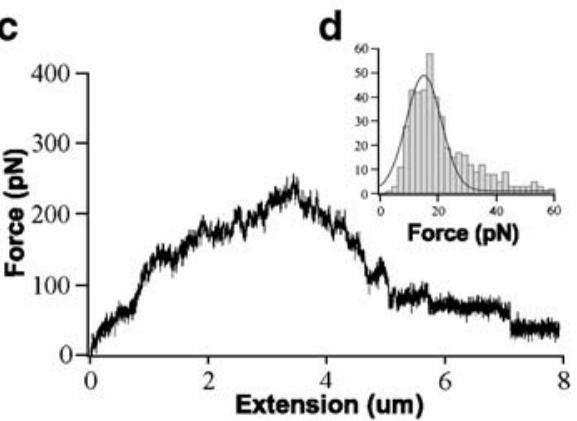

h

g

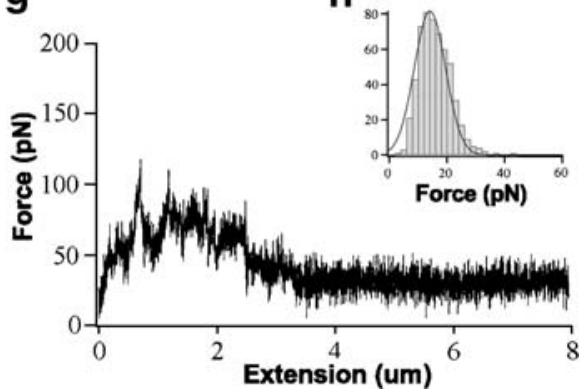

Fig. 2 Local AFM probing of the mechanical profile of CD44 adhesion sites using an HA-decorated tip. The upper and lower panels present the results on resting and PMA-stimulated U-373 GM cells respectively. a, e Superimposed fluorescent micrographs showing the CD44 receptors (green) and actin network (red). The scale bar corresponds to $10 \mu \mathrm{m}$. b, f are mechanical models illustrating the interaction between the CD44 membrane receptor, the actin cytoskeleton, and the HA-functionalised tip before and after PMA stimulation of the cells. c, $\mathbf{g}$ Typical force-elongation curves recorded when the HA tip is retracted from the cell surface. Discontinuities in the curves are associated with molecular rupture events, in which quantification is shown as histograms $(\mathbf{d}, \mathbf{h})$ 
a large glycosaminoglycan found in several tissues where it influences the course of cell migration and invasion [65]. As shown in Fig. 2a, the surfaces of resting cells exhibited microvilli-like structures supported by cortical actin that ensure the direct presentation of CD44 receptors to an extracellular matrix rich in HA. CD44 is the most common membrane receptor for cell binding to HA [66]. A typical force curve (Fig. 2c) shows that final rupture occurs after a large extension (up to several micrometers), indicating that the cell membrane undergoes a major deformation when the HA tip is retracted from the cell surface. In this typical curve, the final rupture is preceded by a constant force plateau, which is a common feature of membrane tethers pulled from the cell surface $[17,26,27,48,67]$. The rupture forces of these membrane tethers are significantly higher $(30-50 \mathrm{pN})$ than those involved in single $\mathrm{HA}-\mathrm{CD} 44$ interactions $(20-30 \mathrm{pN})$. This might define the limit of the force that can be sustained by adhesion sites if the receptors are not strongly anchored to the underlying cytoskeleton. Moreover, membrane are likely to occur if several receptor in close proximity are pulled at the same time. This simple concept runs counter to the need for a strong unimolecular adhesion interaction when a receptor is anchored solely through the lipid membrane. Pulling on HA bounds to the CD44 receptors located over the perinuclear region or at the leading edge, a region that is highly involved in cell motility, does not produce any significant differences in force profiles. This homogeneity in HA adhesion over the entire cell surface is consistent with the resting state of the cells. Figure $2 \mathrm{~b}$ illustrates the results obtained with glioblastoma cells stimulated with phorbol myristate acetate (PMA), a tumour promoter that activates protein kinase $\mathrm{C}$ (PKC). Deregulation of PKC is an important feature in the invasiveness and metastatic behaviour of cancer cells [68]. After stimulation one can clearly observe the reorganisation of the actin network and the relocalisation of the CD44 receptors to the leading edge on fluorescent micrographs (Fig. 2e). In accordance, no adhesion events are detected in the perinuclear region, whereas specific recognition events can be detected at the leading edge of the cells. Interestingly, the magnitude of the rupture force is not significantly influenced by the PMA-induced relocalisation of the CD44 receptors to the leading edge (see histograms before and after PMA stimulation) However, cell membrane deformations, which appear as plateaus in the force-distance retraction curves, are non-existent (Fig. 2g). Furthermore, the final rupture occurs at shorter distances from the original cell surface, an indication of increased resistance to membrane deformation. Hence, the exclusive presence of HA-binding sites at the leading edge, together with an apparent reduced compliance of the cell, is expected to give a clear advantage for the directed progression of cancer cells in a HA-enriched environment. The mechanism for the abrogation of membrane tether creation is unclear and may involve multiple factors, including increased membrane tension and a more cohesive cytoskeletalmembrane network. PKC can cause the phosphorylation of CD44 [69], as well as various cytoskeleton adaptor proteins involved in the anchoring of membrane receptors to the cortical actin [70]. Pulling on adhesion recognition pairs directly on a living cell makes it possible to evaluate the molecular mechanisms involved in the regulation of physical interactions between cytosolic and membraneanchored proteins. As such, monitoring local cell deformations mediated by specific molecular adhesion pairs will refine our understanding of physiological processes that rely on specific interactions between extracellular and intracellular biomolecular structures. A very interesting extension of the technique, which has been reviewed elsewhere [31-34], involves mapping specific molecular entities on the surface of living cells in the so-called force affinity imaging mode. While substantial advances have been made in this area, the possibility of cell membrane deformations might represent a major obstacle to the realisation of precise mapping of recognition events on individual living cells.

\section{Incorporation of biological materials into individual cells}

Current cell biology methods are limited to the characterisation of whole-cell populations. In the future, nanotechnology will be applied in cellular biology to influence and control the homeostasis of individual cells in relation to a group of cells and allow the design of complex cell assemblies. Microcapillary techniques are one of the most commonly used methods to introduce and extract genes, proteins, molecules and other materials into and from individual cells [71, 72]. However, these techniques are not very accurate in terms of spatial displacement and often cause irreversible damage to sensitive cells.

A number of studies have demonstrated that precise, gentle cell nanosurgery using nanoscaled techniques is possible [73-78]. Osada et al. recently showed that endogenous molecules can be extracted from the cytosol of living cells using an AFM tip [73]. They retrieved messenger RNA (mRNA) from a single cell by driving into the cytosol the tip of a standard silicone nitride cantilever routinely used in AFM experiments. The isolated mRNA was quantified by reverse PCR of the $\beta$-actin transcript. Such intrusions into cells are non-lethal [73, 75, 79, 80], which opens up new avenues for monitoring cellular components in real-time. If biomolecules can be retrieved from the cytosol using an AFM tip, it is also possible to insert material into selected cells. Chen et al. [79] introduced 
quantum dots into the cytosol of cells using a carbon nanotube mounted on an AFM tip. The transfer of the material was based on the reversibility of the disulfide-based linker when it came into contact with the cytosolic environment. In another study, an ultra-thin needle fabricated from a commercially available AFM tip was used to inject plasmid DNA into a living cell to modulate gene expression [78]. The authors clearly demonstrated that genetic material could be injected in a controlled manner without causing irreversible cell damage. While their approach was straightforward and reproducible, it was limited by the availability of the microfabricated thin nanoneedles.

Cuerrier et al. showed that single-cell transfections are easily achieved using a commercially available AFM tip on which plasmids encoding for green fluorescent protein (EGFP) are non-specifically adsorbed [80]. Figure 3a illustrates the transfection procedure. The tip is allowed to contact with the cell surface (1) and is then pushed against the surface (2) until it punctures the membrane (3), which is assumed to be a sine qua non condition for delivering the genetic material. This event is monitored in real-time through the deflection of the cantilever as a function of piezo displacement. The success of the transfection is assessed by the expression of fluorescent EGFP. Figures 3b,c correspond to two independent micrographs taken $24 \mathrm{~h}$ after the transfections of single cells. The presence of multiple cells expressing detectable levels of EGFP indicates that the transfected cells remain viable and divide
Fig. 3 Single-cell AFM transfection experiment. a A typical force-distance curve obtained during the spearing of the cell. The tip is located over the cell (1) and then lowered into contact the cell membrane (2) until it penetrates into the cell where the release of the plasmids occurs (3). The asterisk indicates a discontinuity in the force-distance profile corresponding to the puncture of the cell membrane. b-e Superimposed phase contrast and epifluorescence images showing the expression of EGFP in HEK-293 cells after transfection. b, c Images taken $24 \mathrm{~h}$ after two independent single-cell transfections. d, e Subsequent micrographs taken 48 and $72 \mathrm{~h}$, respectively, after the transfection of a single cell. The scale bars correspond to $10 \mu \mathrm{m}(\mathbf{b}, \mathbf{c})$ and $60 \mu \mathrm{m}(\mathbf{d}, \mathbf{e})$
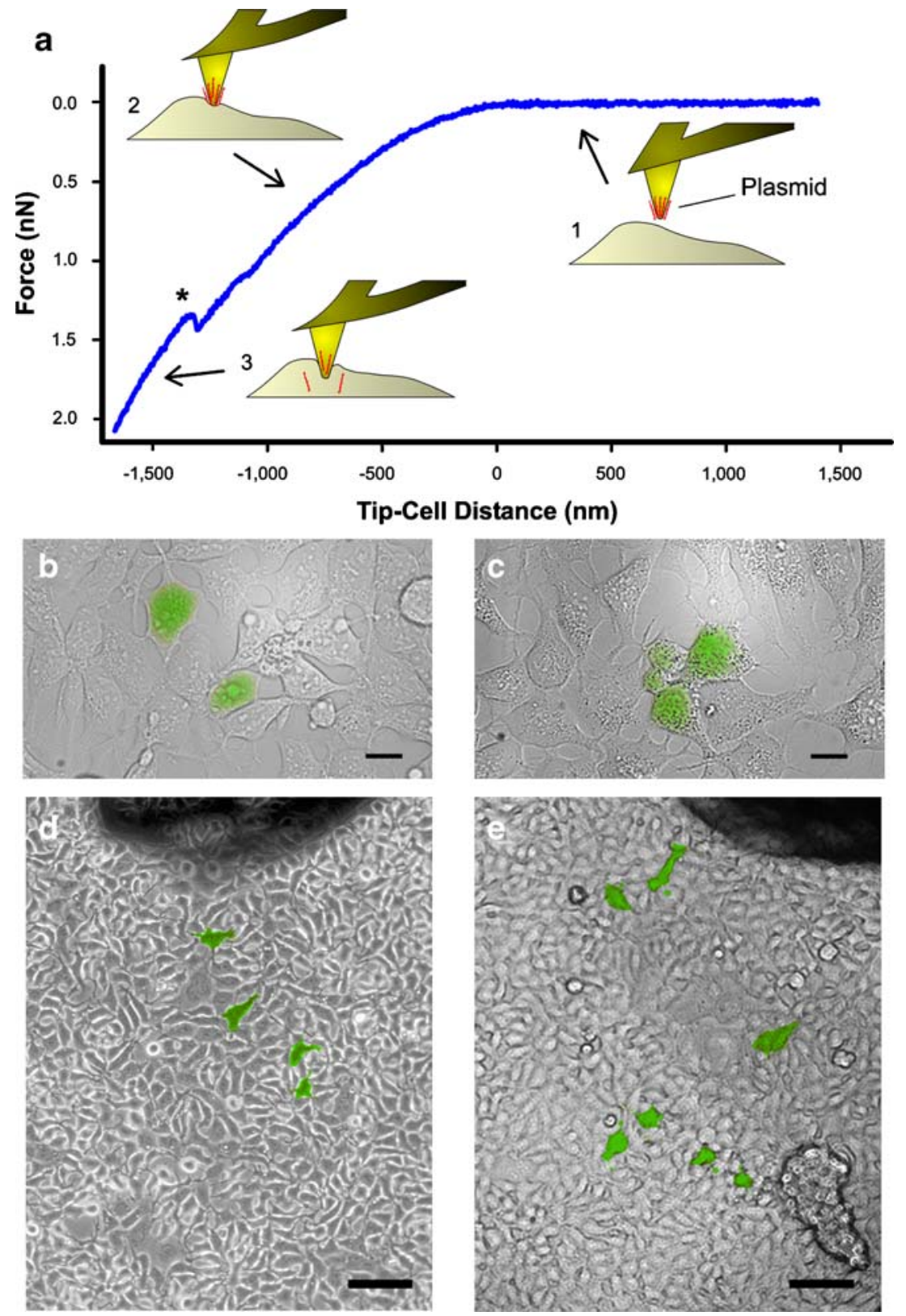
over the 24-h period following the procedure. Cell viability was monitored for an additional 48 and $72 \mathrm{~h}$. Four cells exhibiting similar levels of fluorescence intensity can be observed after $48 \mathrm{~h}$ (Fig. 3d), confirming that the transfected cell grows and divided normally. The same field after $72 \mathrm{~h}$ (Fig. 3e) shows eight cells with similar levels of fluorescence, suggesting that the initially transfected cell has permanently incorporated the genetic material, indicating a stable transfection. Indeed, transient transfection of a single cell would most likely have generated heterogeneous levels of EGFP in the cell population as a whole.

Common to all of these AFM-based nanomanipulations are the low mortality rate and limited physical disturbances of the cell. This technique, thus, provides a new approach for studying gene expression in single mammalian cells with the potential for developing finely tuned cellular and molecular biology models. This would be particularly useful in the study of cell-cell interactions and for experiments that require consecutive transfections of different plasmids into a single cell. In a broader perspective, these approaches will allow the controlled insertion of molecular entities into single living cells with the goal of influencing and controlling specific cell processes leading to the rational design and engineering of novel cellular functionalities.

\section{Perspectives: induction of cellular processes through AFM-based nanomanipulations}

Investigating physiological events at the individual cell level using nanotechnologies will have a major impact on cell research. Topographical AFM images of dynamic cell processes are the best-known results of AFM investigations. A multitude of biological problems at the cell level can be studied by precisely nanomanipulating the AFM tip. The past decade has witnessed the emergence of a large number of AFM applications, including the stretching and unfolding of macromolecules and the recording of molecular recognition rupture events. These two areas of research have been described in great details by others in several excellent reviews $[5,7,8,11,12]$.

Probing of molecular recognition events directly on the surface of a single selected cell provide mechanistic details of cell interactions with the surrounding environment. This approach can also be used to control and modulate cell mechanisms that rely on the biochemical activation of cell membrane receptors. AFM tips decorated with appropriate receptor agonists can be effective tools for specifically controlling and modulating cell processes. AFM force sensing, coupled with optical tracking of induced intracellular signals, is a very appealing method for studying cell responses to localised biochemical or mechanical stimulations. Very localised stimuli at the cell surface often initiate intracellular signals and mechanical responses that are crucial in a number of cell processes such as chemotaxis and focal adhesion assembly. For example, HA can be an important chemoattractant molecule controlling the invasion of cancer cells [81]. Oliferenko et al. have shown that the local application of an HA microgradient to glioblastoma cells induces major lamellipodia outgrowth via a rac-1-dependent signalling pathway [82]. Time-lapse images of cell chemotaxis induced by an HA-decorated AFM tip are shown in Fig. 4. The HAdecorated tip was allowed to come into contact with the cell surface with minimal indention force, then retracted for
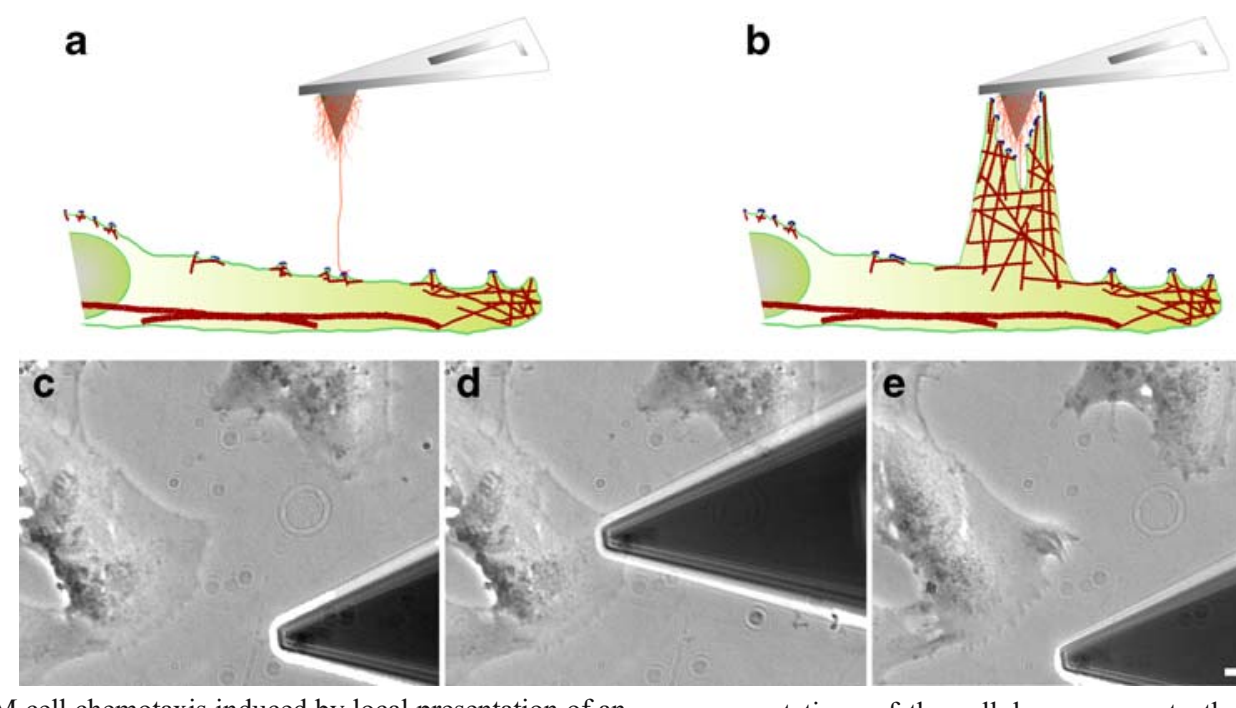

Fig. 4 U-373 GM cell chemotaxis induced by local presentation of an HA-decorated AFM tip. The tip was lowered onto the cell surface to allow the HA molecules to locally bind to the CD44 membrane receptors. The tip was then lifted up by 2 to $3 \mu \mathrm{m}$ while keeping the HA molecular bridge under tension for $30 \mathrm{~min}$. a, b are schematic

representations of the cellular response to the local HA stimulation. c-e Respectively, phase contrast micrographs of the cell before, during and after the contact of the HA tip with the CD44 receptors on the cell surface. The scale bar corresponds to $10 \mu \mathrm{m}$ 
several micrometers and held in this position for $30 \mathrm{~min}$ while preserving the molecular bridge between the HA and the cell surface CD44 receptors (see schematics representations in Figs. 4a,b). This procedure results in significant local structural changes (Fig. 4e) that are most likely driven by cytoskeleton remodelling. These changes can be observed by optical microscopy after displacing the AFM tip from the stimulated area. This experiment demonstrates that it is possible to initiate cell responses by applying a spatially limited biochemical stimulus. One can easily envision the opportunities to stimulate cells while tracking relevant intracellular signals and morphological changes through optical monitoring of fluorescence markers. Furthermore, as demonstrated in the AFM transfection experiments, the incorporation of specific ligands or functional units inside a cell, enzymes for example, could be a powerful way to locally modulate physiological processes. The various AFM applications presented in this review point to the enormous potential of AFM in advancing our knowledge of cell biology and physiology.

\section{References}

1. Engel A, Muller DJ (2000) Observing single biomolecules at work with the atomic force microscope. Nat Struct Biol 7:715-718

2. Milhiet PE, Giocondi MC, Le Grimellec C (2003) AFM imaging of lipid domains in model membranes. Sci World J 3:59-74

3. Scheuring S, Sturgis JN, Prima V, Bernadac A, Levy D, Rigaud JL (2004) Watching the photosynthetic apparatus in native membranes. Proc Natl Acad Sci U S A 101:11293-11297

4. Maco B, Fahrenkrog B, Huang NP, Aebi U (2006) Nuclear pore complex structure and plasticity revealed by electron and atomic force microscopy. Methods Mol Biol 322:273-288

5. Muller DJ, Sapra KT, Scheuring S, Kedrov A, Frederix PL, Fotiadis D, Engel A (2006) Single-molecule studies of membrane proteins. Curr Opin Struct Biol 16:489-495

6. Rief M, Pascual J, Saraste M, Gaub HE (1999) Single molecule force spectroscopy of spectrin repeats: low unfolding forces in helix bundles. J Mol Biol 286:553-561

7. Fisher TE, Marszalek PE, Fernandez JM (2000) Stretching single molecules into novel conformations using the atomic force microscope. Nat Struct Biol 7:719-724

8. Wang K, Forbes JG, Jin AJ (2001) Single molecule measurements of titin elasticity. Prog Biophys Mol Biol 77:1-44

9. Marshall BT, Long M, Piper JW, Yago T, McEver RP, Zhu C (2003) Direct observation of catch bonds involving cell-adhesion molecules. Nature 423:190-193

10. Kienberger F, Ebner A, Gruber HJ, Hinterdorfer P (2006) Molecular recognition imaging and force spectroscopy of single biomolecules. Acc Chem Res 39:29-36

11. Lee CK, Wang YM, Huang LS, Lin S (2007) Atomic force microscopy: determination of unbinding force, off rate and energy barrier for protein-ligand interaction. Micron 38:446-461

12. Robert P, Benoliel AM, Pierres A, Bongrand P (2007) What is the biological relevance of the specific bond properties revealed by single-molecule studies? J Mol Recognit (in press)

13. Thie M, Rospel R, Dettmann W, Benoit M, Ludwig M, Gaub HE, Denker HW (1998) Interactions between trophoblast and uterine epithelium: monitoring of adhesive forces. Hum Reprod 13:32113219

14. Lehenkari PP, Horton MA (1999) Single integrin molecule adhesion forces in intact cells measured by atomic force microscopy. Biochem Biophys Res Commun 259:645-650

15. Sagvolden G, Giaever I, Pettersen EO, Feder J (1999) Cell adhesion force microscopy. Proc Natl Acad Sci U S A 96:471-476

16. Benoit M, Gabriel D, Gerisch G, Gaub HE (2000) Discrete interactions in cell adhesion measured by single-molecule force spectroscopy. Nat Cell Biol 2:313-317

17. Benoit M, Gaub HE (2002) Measuring cell adhesion forces with the atomic force microscope at the molecular level. Cells Tissues Organs 172:174-189

18. Zhang X, Wojcikiewicz E, Moy VT (2002) Force spectroscopy of the leukocyte function-associated antigen-1/intercellular adhesion molecule-1 interaction. Biophys J 83:2270-2279

19. Simon A, Durrieu MC (2006) Strategies and results of atomic force microscopy in the study of cellular adhesion. Micron 37: $1-13$

20. Wojcikiewicz EP, Zhang X, Chen A, Moy VT (2003) Contributions of molecular binding events and cellular compliance to the modulation of leukocyte adhesion. J Cell Sci 116:2531-2539

21. Lee I, Marchant RE (2003) Molecular interaction studies of hemostasis: fibrinogen ligand-human platelet receptor interactions. Ultramicroscopy 97:341-352

22. Hanley WD, Wirtz D, Konstantopoulos K (2004) Distinct kinetic and mechanical properties govern selectin-leukocyte interactions. J Cell Sci 117:2503-2511

23. Zhang X, Chen A, De Leon D, Li H, Noiri E, Moy VT, Goligorsky MS (2004) Atomic force microscopy measurement of leukocyte-endothelial interaction. Am J Physiol Heart Circ Physiol 286:H359-367

24. Wojcikiewicz EP, Zhang X, Moy VT (2004) Force and compliance measurements on living cells using atomic force microscopy (AFM). Biol Proced Online 6:1-9

25. Chang MI, Panorchan P, Dobrowsky TM, Tseng Y, Wirtz D (2005) Single-molecule analysis of human immunodeficiency virus type 1 gp120-receptor interactions in living cells. J Virol 79: $14748-14755$

26. Puech PH, Taubenberger A, Ulrich F, Krieg M, Muller DJ, Heisenberg CP (2005) Measuring cell adhesion forces of primary gastrulating cells from zebrafish using atomic force microscopy. J Cell Sci 118:4199-4206

27. Afrin R, Ikai A (2006) Force profiles of protein pulling with or without cytoskeletal links studied by AFM. Biochem Biophys Res Commun 348:238-244

28. Puech PH, Poole K, Knebel D, Muller DJ (2006) A new technical approach to quantify cell-cell adhesion forces by AFM. Ultramicroscopy 106:637-644

29. Yang H, Yu J, Fu G, Shi X, Xiao L, Chen Y, Fang X, He C (2007) Interaction between single molecules of Mac-1 and ICAM-1 in living cells: An atomic force microscopy study. Exp Cell Res 313:3497-3504

30. Lamontagne CA, Grandbois M (2008) PKC-induced stiffening of hyaluronan/CD44 linkage; local force measurements on glioma cells. Exp Cell Res 314:227-236

31. Dupres V, Verbelen C, Dufrene YF (2007) Probing molecular recognition sites on biosurfaces using AFM. Biomaterials 28: 2393-2402

32. Hinterdorfer P, Dufrene YF (2006) Detection and localization of single molecular recognition events using atomic force microscopy. Nat Methods 3:347-355

33. Dufrene YF (2003) Recent progress in the application of atomic force microscopy imaging and force spectroscopy to microbiology. Curr Opin Microbiol 6:317-323 
34. Horton M, Charras G, Lehenkari P (2002) Analysis of ligandreceptor interactions in cells by atomic force microscopy. J Recept Signal Transduct Res 22:169-190

35. Wu HW, Kuhn T, Moy VT (1998) Mechanical properties of L929 cells measured by atomic force microscopy: effects of anticytoskeletal drugs and membrane crosslinking. Scanning 20:389-397

36. A-Hassan E, Heinz WF, Antonik MD, D'Costa NP, Nageswaran S, Schoenenberger CA, Hoh JH (1998) Relative microelastic mapping of living cells by atomic force microscopy. Biophys J 74:15641578

37. Rotsch C, Jacobson K, Radmacher M (1999) Dimensional and mechanical dynamics of active and stable edges in motile fibroblasts investigated by using atomic force microscopy. Proc Natl Acad Sci U S A 96:921-926

38. Kasas S, Wang X, Hirling H, Marsault R, Huni B, Yersin A, Regazzi R, Grenningloh G, Riederer B, Forro L et al (2005) Superficial and deep changes of cellular mechanical properties following cytoskeleton disassembly. Cell Motil Cytoskeleton 62: 124-132

39. Radmacher M (2007) Studying the mechanics of cellular processes by atomic force microscopy. Methods Cell Biol 83:347-372

40. Kuznetsova TG, Starodubtseva MN, Yegorenkov NI, Chizhik SA, Zhdanov RI (2007) Atomic force microscopy probing of cell elasticity. Micron 38:824-833

41. Langer MG, Fink S, Koitschev A, Rexhausen U, Horber JK, Ruppersberg JP (2001) Lateral mechanical coupling of stereocilia in cochlear hair bundles. Biophys J 80:2608-2621

42. Charras GT, Lehenkari PP, Horton MA (2001) Atomic force microscopy can be used to mechanically stimulate osteoblasts and evaluate cellular strain distributions. Ultramicroscopy 86:85-95

43. Stauss HM (2002) Baroreceptor reflex function. Am J Physiol Regul Integr Comp Physiol 283:R284-286

44. Li S, Huang NF, Hsu S (2005) Mechanotransduction in endothelial cell migration. J Cell Biochem 96:1110-1126

45. Prass M, Jacobson K, Mogilner A, Radmacher M (2006) Direct measurement of the lamellipodial protrusive force in a migrating cell. J Cell Biol 174:767-772

46. Vollrath MA, Kwan KY, Corey DP (2007) The micromachinery of mechanotransduction in hair cells. Annu Rev Neurosci 30:339-365

47. Poole K, Meder D, Simons K, Muller D (2004) The effect of raft lipid depletion on microvilli formation in MDCK cells, visualized by atomic force microscopy. FEBS Lett 565:53-58

48. Sun M, Graham JS, Hegedus B, Marga F, Zhang Y, Forgacs G, Grandbois M (2005) Multiple membrane tethers probed by atomic force microscopy. Biophys J 89:4320-4329

49. Ye HQ, Gan L, Yang XL, Xu HB (2006) Membrane-associated cytotoxicity induced by realgar in promyelocytic leukemia HL-60 cells. J Ethnopharmacol 103:366-371

50. Frankel DJ, Pfeiffer JR, Surviladze Z, Johnson AE, Oliver JM, Wilson BS, Burns AR (2006) Revealing the topography of cellular membrane domains by combined atomic force microscopy/ fluorescence imaging. Biophys J 90:2404-2413

51. Oberleithner H, Riethmuller C, Ludwig T, Shahin V, Stock C, Schwab A, Hausberg M, Kusche K, Schillers H (2006) Differential action of steroid hormones on human endothelium. J Cell Sci 119: 1926-1932

52. Shroff SG, Saner DR, Lal R (1995) Dynamic micromechanical properties of cultured rat atrial myocytes measured by atomic force microscopy. Am J Physiol 269:C286-292

53. Domke J, Parak WJ, George M, Gaub HE, Radmacher M (1999) Mapping the mechanical pulse of single cardiomyocytes with the atomic force microscope. Eur Biophys J 28:179-186

54. Pelling AE, Veraitch FS, Pui-Kei Chu C, Nicholls BM, Hemsley AL, Mason C, Horton MA (2007) Mapping correlated membrane pulsations and fluctuations in human cells. J Mol Recognit (in press)
55. Small J, Rottner K, Hahne P, Anderson KI (1999) Visualising the actin cytoskeleton. Microsc Res Tech 47:3-17

56. Zicha D, Dobbie IM, Holt MR, Monypenny J, Soong DY, Gray C, Dunn GA (2003) Rapid actin transport during cell protrusion. Science 300:142-145

57. Pelling AE, Sehati S, Gralla EB, Valentine JS, Gimzewski JK (2004) Local nanomechanical motion of the cell wall of Saccharomyces cerevisiae. Science 305:1147-1150

58. Pelling AE, Sehati S, Gralla EB, Gimzewski JK (2005) Time dependence of the frequency and amplitude of the local nanomechanical motion of yeast. Nanomedicine 1:178-183

59. Szabo B, Selmeczi D, Kornyei Z, Madarasz E, Rozlosnik N (2002) Atomic force microscopy of height fluctuations of fibroblast cells. Phys Rev E Stat Nonlin Soft Matter Phys 65: 041910

60. Auger-Messier M, Turgeon ES, Leduc R, Escher E, Guillemette G (2005) The constitutively active N111G-AT1 receptor for angiotensin II modifies the morphology and cytoskeletal organization of HEK-293 cells. Exp Cell Res 308:188-195

61. Mathur AB, Truskey GA, Reichert WM (2000) Total internal reflection microscopy and atomic force microscopy (TIRFMAFM) to study stress transduction mechanisms in endothelial cells. Crit Rev Biomed Eng 28:197-202

62. Charras GT, Horton MA (2002) Single cell mechanotransduction and its modulation analyzed by atomic force microscope indentation. Biophys J 82:2970-2981

63. Thie M, Denker HW (2002) In vitro studies on endometrial adhesiveness for trophoblast: cellular dynamics in uterine epithelial cells. Cells Tissues Organs 172:237-252

64. Zaidel-Bar R, Cohen M, Addadi L, Geiger B (2004) Hierarchical assembly of cell-matrix adhesion complexes. Biochem Soc Trans $32: 416-420$

65. Bellail AC, Hunter SB, Brat DJ, Tan C, Van Meir EG (2004) Microregional extracellular matrix heterogeneity in brain modulates glioma cell invasion. Int J Biochem Cell Biol 36:1046-1069

66. Isacke CM, Yarwood H (2002) The hyaluronan receptor, CD44. Int J Biochem Cell Biol 34:718-721

67. Hosu BG, Sun M, Marga F, Grandbois M, Forgacs G (2007) Eukaryotic membrane tethers revisited using magnetic tweezers. Phys Biol 4:67-78

68. Griner EM, Kazanietz MG (2007) Protein kinase C and other diacylglycerol effectors in cancer. Nat Rev Cancer 7:281-294

69. Kalomiris EL, Bourguignon LY (1989) Lymphoma protein kinase $\mathrm{C}$ is associated with the transmembrane glycoprotein, GP85, and may function in GP85-ankyrin binding. J Biol Chem 264:81138119

70. Larsson C (2006) Protein kinase $C$ and the regulation of the actin cytoskeleton. Cell Signal 18:276-284

71. Tsulaia TV, Prokopishyn NL, Yao A, Carsrud ND, Carou MC, Brown DB, Davis BR, Yannariello-Brown J (2003) Glass needlemediated microinjection of macromolecules and transgenes into primary human mesenchymal stem cells. J Biomed Sci 10:328336

72. King R (2004) Gene delivery to mammalian cells by microinjection. Methods Mol Biol 245:167-174

73. Osada T, Uehara H, Kim H, Ikai A (2003) mRNA analysis of single living cells. J Nanobiotechnology 1:2

74. Hategan A, Law R, Kahn S, Discher DE (2003) Adhesively tensed cell membranes: lysis kinetics and atomic force microscopy probing. Biophys J 85:2746-2759

75. Uehara H, Osada T, Ikai A (2004) Quantitative measurement of mRNA at different loci within an individual living cell. Ultramicroscopy 100:197-201

76. Obataya I, Nakamura C, Han S, Nakamura N, Miyake J (2005) Nanoscale operation of a living cell using an atomic force microscope with a nanoneedle. Nano Lett 5:27-30 
77. Han SW, Nakamura C, Obataya I, Nakamura N, Miyake J (2005) A molecular delivery system by using AFM and nanoneedle. Biosens Bioelectron 20:2120-2125

78. Han S, Nakamura C, Obataya I, Nakamura N, Miyake J (2005) Gene expression using an ultrathin needle enabling accurate displacement and low invasiveness. Biochem Biophys Res Commun 332:633-639

79. Chen X, Kis A, Zettl A, Bertozzi CR (2007) A cell nanoinjector based on carbon nanotubes. Proc Natl Acad Sci U S A 104:82188222
80. Cuerrier CM, Lebel R, Grandbois M (2007) Single cell transfection using plasmid decorated AFM probes. Biochem Biophys Res Commun 355:632-636

81. Tzircotis G, Thorne RF, Isacke CM (2005) Chemotaxis towards hyaluronan is dependent on CD44 expression and modulated by cell type variation in CD44-hyaluronan binding. J Cell Sci 118:5119-5128

82. Oliferenko S, Kaverina I, Small JV, Huber LA (2000) Hyaluronic acid (HA) binding to CD44 activates Rac1 and induces lamellipodia outgrowth. J Cell Biol 148:1159-1164 